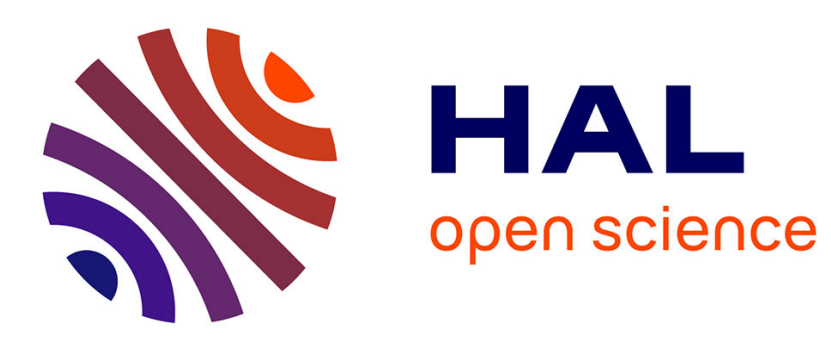

\title{
Enhancing power grid synchronization through time delayed feedback control of solitary states
}

\author{
Halgurd Taher, Simona Olmi, Eckehard Schöll
}

\section{To cite this version:}

Halgurd Taher, Simona Olmi, Eckehard Schöll. Enhancing power grid synchronization through time delayed feedback control of solitary states. ECC 2020 - European Control Conference, May 2020, Saint Petersburg, Russia. pp.1981-1986, 10.23919/ECC51009.2020.9143594 . hal-02972574

\section{HAL Id: hal-02972574 \\ https://hal.inria.fr/hal-02972574}

Submitted on 24 Nov 2020

HAL is a multi-disciplinary open access archive for the deposit and dissemination of scientific research documents, whether they are published or not. The documents may come from teaching and research institutions in France or abroad, or from public or private research centers.
L'archive ouverte pluridisciplinaire HAL, est destinée au dépôt et à la diffusion de documents scientifiques de niveau recherche, publiés ou non, émanant des établissements d'enseignement et de recherche français ou étrangers, des laboratoires publics ou privés. 


\title{
Enhancing power grid synchronization through time delayed feedback control of solitary states
}

\author{
Halgurd Taher, ${ }^{1,2}$ Simona Olmi, ${ }^{1,3, *}$ and Eckehard Schöll ${ }^{2}$ \\ ${ }^{1}$ Inria Sophia Antipolis Méditerranée Research Centre, 2004 Route des Lucioles, 06902 Valbonne, France \\ ${ }^{2}$ Institut für Theoretische Physik, Technische Universität Berlin, Hardenbergstraße 36, 10623 Berlin, Germany \\ ${ }^{3}$ CNR - Consiglio Nazionale delle Ricerche - Istituto dei Sistemi Complessi, 50019, Sesto Fiorentino, Italy
}

We study the synchronization and stability of power grids within the Kuramoto phase oscillator model with inertia with a bimodal frequency distribution representing the generators and the loads. We identify critical nodes through solitary frequency deviations and Lyapunov vectors corresponding to unstable Lyapunov exponents. To cure dangerous deviations from synchronization we propose timedelayed feedback control, which is an efficient control concept in nonlinear dynamic systems. Different control strategies are tested and compared with respect to the minimum number of controlled nodes required to achieve synchronization and Lyapunov stability. As a proof of principle, this fast-acting control method is demonstrated using a model of the German power transmission grid.

Introduction. Investigations of synchronization phenomena in nonlinear dynamical networks are of major interest with respect to a wide field of applications in natural and technological systems [1, 2], e.g., neural networks in the human brain, or supply and communication networks and power grids, which naturally have a strong link to economy. Research in these fields has revealed diverse phenomena related to synchronization, ranging from partial synchronization patterns to asynchronous states [3-5]. In particular, scenarios leading from full synchronization to asynchronicity via solitary states, i.e., single nodes which are desynchronized from the rest, play an important role for complex dynamical systems [6, 7], and in this work we will show that they are fundamental also for power grids.

Infrastructure, e.g., public transportation, medical care and a vast number of other everyday life applications, rely on electrical power supply. Given the fact that modern power transmission grids, notably if they include renewable energy sources, differ significantly from conventional power grids with regards to topology and local dynamics [8-10], it is necessary to identify, understand, and cure the arising challenges and problems. In particular, malfunctioning grids can be the result of power outages, which occur for various reasons, including line overload or voltage collapse. Here we will focus on the loss of synchrony. In normal operation, a power grid runs in the synchronous state in which all frequencies equal the nominal frequency $(50$ or $60 \mathrm{~Hz}$ ) and in which steady power flows balance supply and demand at all nodes. When parts of a power grid desynchronize, destructive power oscillations emerge. To avoid damage, affected components must then be switched off. However, such switchings can in turn desynchronize other grid components, possibly provoking a cascade of further shut-downs and ending in a largescale blackout [11-13].

The failure of a transmission line during a blackout can be determined not only by the network topology and the static distribution of electric flow but also by the collective transient dynamics of the entire system where the time scale of system instabilities is of seconds $[14,15]$. In general, grids are designed such that the synchronous state is locally stable, implying that a cascade-triggering desynchronization cannot be caused by a small perturbation. However, even if the synchronous state is stable against small perturbations, the state space of power grids is also populated by numerous stable non-synchronous states to which the grid might be driven by short circuits, fluctuations in renewable energy generation or other large perturbations [14, 16, 17]. Therefore it is of fundamental interest to explore the relation between network properties and grid stability against large perturbations $[14,18,19]$. Yet many intriguing questions on the relation between grid topology and local stability are still not understood, and this is a highly active field of research. Decentralized grids tend to be less robust with respect to dynamical perturbations, but more robust against structural perturbations to the grid topology [20]. However, adding new links may not only promote but also destroy synchrony, thus inducing power outages when geometric frustration occurs $[21,22]$. The local stability can be improved by relating the specifics of the dynamical units and the network structure [23-25], or predicting a priori which links are critical via the link's redundant capacity and a renormalized response theory [26].

In the following we will demonstrate the role played by the solitary nodes in driving the populations out of synchrony and the necessity to control these nodes when restoring both stability and synchronization. Solitary nodes can be related to local instabilities via the application of a standard stability toolbox (i.e., Lyapunov exponents and Lyapunov vectors), and to topological properties of the network, like dead ends, thus complementing the analysis reported in [25]. In particular, once we have identified the critical power grid nodes which undermine stability and synchronization, we will apply time-delayed feedback control to a small subset of these nodes, in order to cure a desynchronized and unstable power grid. Time-delayed feedback is an efficient mechanism known in nonlinear dynamics and often used to control unstable systems [27, 28]. Generator and consumer dynamics will be described in terms of Kuramoto oscillators with 
inertia, firstly introduced in [29] to map an electrical grid, connected by transmission lines, to a network of coupled rotators. As a specific example, we consider the topology of the German ultra-high voltage power transmission grid $(220 \mathrm{kV}$ and $380 \mathrm{kV}$ ).

The Kuramoto model with inertia. The Kuramoto model with inertia describes the phase and frequency dynamics of $N$ coupled synchronous machines, i.e., generators or consumers within the power grid, where mechanical and electrical phase and frequency are assumed to be identical. The $N$ dynamic equations are

$$
\ddot{\theta}_{i}+\alpha \dot{\theta}_{i}=\frac{P_{i}}{I_{i} \omega_{G}}+\frac{K_{i}}{I_{i} \omega_{G}} \sum_{j=1}^{N} A_{i j} \sin \left(\theta_{j}-\theta_{i}\right),
$$

with the phase $\theta_{i}(t)$ and frequency $\dot{\theta}_{i}(t)=\frac{d \theta_{i}}{d t}$ of node $i=1, \ldots, N$. Both dynamic variables $\theta_{i}(t), \dot{\theta}_{i}(t)$ are defined relative to a frame rotating with the reference power line frequency $\omega_{G}$, e.g., $50 \mathrm{~Hz}$ for the European transmission grid. The distribution of net power generation $\left(P_{i}>0\right)$ and consumption $\left(P_{i}<0\right)$ is bimodal; it corresponds to the inherent frequency distribution in the nondimensionalized Kuramoto model. The power balance requires $\sum_{i} P_{i}=0$. We assume homogeneously distributed transmission capacities $K_{i}=K$. Thus $K$ can be regarded as the network coupling strength. The adjacency matrix $A$ takes values 1 if node $i$ has a transmission line connected to node $j$, and 0 otherwise. The connectivity graph is undirected. Moreover $\alpha$ is the dissipation parameter and takes typical values of $0.1-1 \mathrm{~s}^{-1}[25,30]$. Finally, the moment of inertia $I_{i}$ of turbine $i$ will be set to $I_{i}=I=40 \times 10^{3} \mathrm{~kg} \mathrm{~m}^{2}$, corresponding to generation capacities of a single power plant equal to $400 \mathrm{MW}[25,31]$. With the above definitions, the frequency synchronization criterion reads $\dot{\theta}_{i}(t)=0, \quad \forall i=1, \ldots \mathrm{N}$, i.e., deviations from the reference frequency are zero. In our numerical example we extract the topology $A_{i j}$ from the freely available Open Source Electricity Model for Germany (elmod-de) [32], which describes the German ultra-high voltage transmission grid using $N=438$ nodes connected by 662 transmission lines (see Fig. 1a).

In many previous studies using the Kuramoto model with inertia to model power grid networks, the distribution of net power generation and consumption $P_{i}$ is set to be a bimodal $\delta$-distribution $[20,21,25,33-35]$. Such a distribution assumes homogeneous power generation $P_{i}=P_{\mathcal{G}}>0$ for generators $i \in \mathcal{G}$ and power consumption $P_{i}=-P_{\mathcal{C}}<0$ for consumers $i \in \mathcal{C}$, meaning that all generators and consumers are identical with respect to their power generation and consumption, respectively. Here we consider more complex distributions: first of all, an artificial bimodal Gaussian distribution $P^{\mathrm{G}}[36,37]$ is generated, whose probability density function $p(P)$ is given by the superposition of two Gaussians centered at $\pm P_{0}$ with standard deviation $\sigma$

$$
p(P)=\frac{1}{2 \sigma \sqrt{2 \pi}}\left(e^{-\frac{\left(P-P_{0}\right)^{2}}{2 \sigma^{2}}}+e^{-\frac{\left(P+P_{0}\right)^{2}}{2 \sigma^{2}}}\right) .
$$

Figure $1 \mathrm{~b}$ shows a histogram of the realization $P^{\mathrm{G}}$ used in the numerical simulations of this study. The second distribution $P^{\mathrm{R}}$ shown in Figure $1 \mathrm{c}$ is calculated based on data provided by elmod-de [32] and will be referred to as realworld distribution.

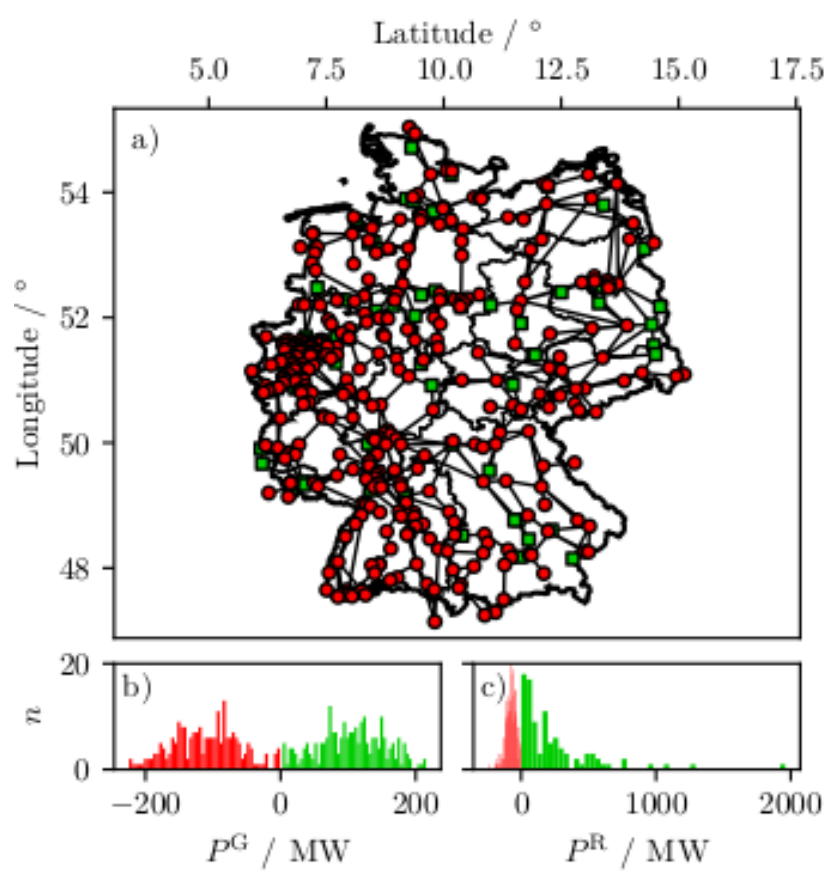

FIG. 1. (a) Map of the German ultra-high voltage power grid, consisting of 95 net generators (green squares) and 343 net consumers (red dots) connected by 662 transmission lines (black lines). (b) Histogram shows a realization of an artificial bimodal Gaussian distribution of natural frequencies; $P_{0}=105 \mathrm{MW}$, $\sigma=P_{0} / 2$. (c) Histogram shows a distribution of natural frequencies based on the German power grid [32]. The green (red) bars correspond to generators (consumers).

Parameter choice. Eq. 1 describes the time evolution of the phase $\theta_{i}(t)$ and frequency $\dot{\theta}_{i}(t)=\frac{d \theta_{i}}{d t}$ of node $i=1, \ldots, N$. In particular $\alpha$ represents the dissipation parameter and takes typical values of $0.1-1 \mathrm{~s}^{-1}[25,30]$. However, in a realistic power grid there are additional sources of dissipation, especially Ohmic losses, and losses caused by damper windings [30], which are not taken into account directly in the coupled oscillator model. Therefore, for this parameter we have chosen slightly higher values: $\alpha=5 / 6 \mathrm{~s}^{-1}$ when a bimodal Gaussian distribution is considered and $\alpha=2 \mathrm{~s}^{-1}$ when the real-world distribution is taken into account to describe the distribution of the net power $P_{i}$. Different dissipation values are necessary for the different distributions in order to obtain comparable setups, i.e., unstable, partially synchronized states at comparable coupling strengths, $K=819 \mathrm{MW}$ for the bimodal Gaussian distribution and $K=729 \mathrm{MW}$ for the real-world one.

Power transmission capacities and moments of inertia were set homogeneously throughout the grid, even though a more realistic approach would suppose a heterogeneous distribution. However the goal of the present paper is to gain insight 
into the principal behavior of large power grids depending on the network topology, and their capability to synchronize by controlling a minimal set of nodes and, for a proof of principle of our control approach, the choice of homogeneous distribution suffices. The choice of using simplified homogeneous transmission line capacities and moments of inertia turned out to be a good compromise when using heterogeneous power distributions, whose realistic values were the only available ones in the open data source.

Macroscopic indicators and Lyapunov analysis. We consider a scenario where, due to an arbitrary dynamical perturbation, some critical nodes have become desynchronized, where we define as critical those nodes withstanding selforganized resynchronization. Synchronization is first gained by performing an adiabatic transition from the asynchronous to the synchronized state for increasing coupling constant: starting with random initial conditions $\theta_{i}(0) \in[-2 \pi, 2 \pi)$, $\dot{\theta}_{i}(0) \in[-1,1)$ at $K=0$, the coupling strength $K$ is increased adiabatically up to $K_{\text {Max }}$ where the system shows synchronized behavior. For each investigated value of $K$, the system is initialized with the final conditions found for the previous coupling value, then the system evolves for a transient time $T_{A}$, such that it can reach a steady state. After the transient time $T_{A}$, characteristic measures are calculated in order to assess the quality of synchronization and the stability of the underlying state $\left\{\theta_{i}\left(T_{A}\right), \dot{\theta}_{i}\left(T_{A}\right)\right\}$. In particular the time-averaged phase velocity profile $\left\langle\omega_{i}\right\rangle_{t} \equiv\left\langle\dot{\theta}_{i}\right\rangle_{t}$ provides information on frequency synchronization of individual nodes $i$, whereas the standard deviation of frequencies $\Delta \omega(t) \equiv \frac{1}{N} \sqrt{\sum_{i=1}^{N}\left(\omega_{i}(t)-\bar{\omega}(t)\right)^{2}}$, is used to estimate the deviation from complete frequency synchronization $(\bar{\omega}(t)$ indicates the instantaneous average grid frequency). In particular $\Delta \omega(t)=0$ when frequency synchronization is attained.

Once a desired synchronized state is reached, a perturbation can occur leading the state out of synchrony. In this situation the overall stability of the power grid might be lost, therefore it is necessary to analyze the time-evolution of small dynamic perturbations $\delta \theta_{i}:=\theta_{i}^{*}-\theta_{i}$ around the steady state $\theta_{i}^{*}$, whose dynamics is ruled by the linearization of Eq. (1) as follows

$$
\delta \ddot{\theta}_{i}+\alpha \delta \dot{\theta}_{i}=\frac{K}{I \omega_{G}} \sum_{j=1}^{N} A_{i j} \cos \left(\theta_{j}-\theta_{i}\right)\left(\delta \theta_{j}-\delta \theta_{i}\right)
$$

The exponential growth rates of the infinitesimal perturbations are measured in term of the associated Lyapunov spectrum $\left\{\lambda_{k}\right\}$, with $k=1, \ldots, 2 N$, numerically estimated by employing the method developed by Benettin et al. [38]. In a dissipative system one has a fixed point whenever the maximal Lyapunov is negative, a periodic motion if $\lambda_{1}=0$ and $\lambda_{2}<0$, a quasi-periodic motion on a Torus $T^{N}$ if $\lambda_{1}=\lambda_{2}=\ldots=\lambda_{N}=0$ and a chaotic motion if the maximal Lyapunov is positive. Therefore whenever $\lambda_{1}>0$, the synchronization looses stability and the system is Lyapunov unstable. In particular one should consider for each Lya- punov exponent $\lambda_{k}$ the corresponding $2 \mathrm{~N}$-dimensional tangent vector $\mathcal{T}^{(k)}=\left(\delta \dot{\theta}_{1}, \ldots, \delta \dot{\theta}_{N}, \delta \theta_{1}, \ldots \delta \theta_{N}\right)$ whose time evolution is given by Eq. (3). Important information about the sources of instability and, in particular, about the oscillators that are more actively contributing to the chaotic dynamics, can be gained by calculating the time averaged evolution of the tangent vector $\mathcal{T}^{(1)}$, here referred to as maximum Lyapunov vector. The Euclidean norm of each $\left\{\theta_{i}, \dot{\theta}_{i}\right\}$ pair in $\mathcal{T}^{(1)}$, averaged in time, is measured for each oscillator as $\xi_{i}:=\left\langle\sqrt{\left[\delta \theta_{i}(t)\right]^{2}+\left[\delta \dot{\theta}_{i}(t)\right]^{2}}\right\rangle_{t}$, once the Lyapunov vector is orthonormalized, i.e. $\left\|\mathcal{T}^{(1)}\right\|=1$. Lyapunov vectors describe the characteristic expanding and contracting directions of a dynamical system. In particular they point in the directions in which an infinitesimal perturbation will grow asymptotically, exponentially at an average rate given by the Lyapunov exponents. When expanded in terms of Lyapunov vectors, a perturbation asymptotically aligns with "the maximum" vector, i.e., the Lyapunov vector in that expansion corresponding to the largest Lyapunov exponent, as this direction outgrows all others. Therefore almost all perturbations align asymptotically with the Lyapunov vector corresponding to the largest Lyapunov exponent in the system [39].

Time-delayed feedback control. In order to enhance frequency synchronization and stability when the system is subject to a dynamical perturbation, the Kuramoto model with inertia is extended by time-delayed feedback control which is an efficient control concept, well known in nonlinear dynamic systems [27, 28], but not commonly employed in power grid engineering [40-45]:

$$
\begin{aligned}
\ddot{\theta}_{i}+\alpha \dot{\theta}_{i} & =\frac{P_{i}}{I \omega_{\mathrm{G}}}+\frac{K}{I \omega_{\mathrm{G}}} \sum_{j=1}^{N} A_{i j} \sin \left(\theta_{j}-\theta_{i}\right) \\
& -\frac{g_{i} \alpha}{\tau}\left[\theta_{i}(t)-\theta_{i}(t-\tau)\right],
\end{aligned}
$$

where $g_{i}$ is the control gain of node $i$ and $\tau$ is the delay time. While primary control sets in to stabilize the frequency and to prevent a large drop within few seconds after a shortage, secondary control is necessary to restore the frequency back to its nominal value of 50 or $60 \mathrm{Hertz}$ within few minutes. On the other hand, our delayed feedback control is able both to stabilize the frequency of the power plant at the origin of the perturbation and to restore the frequency back to its nominal value within few seconds. It turns out that the control is robust against changes in the parameters $\tau, g_{i}$, as shown in [45]. In particular control performance at large enough gain is comparable for a wide range of delay times, therefore, without loss of generality we have chosen $\tau=4$ and $g=1$, being the chosen delay time within this range. Finally it is worth highlighting that the control scheme is applied to a subset ot generators, chosen according to suitably designed strategies. The possibility to control few nodes out of a big network makes the stabilization of the network faster: the control in principle can be switched on for all emerging critical nodes and it turns off automatically when 
the node is synchronized again. Since the control vanishes at full synchronization, it does not double the dissipation rate permanently.

Emergence of solitary states. Figure 2 shows the timeaveraged standard frequency deviation $\langle\Delta \omega\rangle_{t}$ and the maximum Lyapunov exponent $\lambda_{1}$ for each value $K$ of the adiabatic increase for the bimodal Gaussian (panel a) and for the real-world distribution (panel b). Complete frequency synchronization with $\langle\Delta \omega\rangle_{t}=0, \lambda_{1}=0$ is achieved at $K \geq 1320 \mathrm{MW}(K \geq 4200 \mathrm{MW})$ for $P^{\mathrm{G}}\left(P^{\mathrm{R}}\right)$. At smaller coupling strength values asynchronous equilibria with finite values of $\langle\Delta \omega\rangle_{t}$ coexist with the desired synchronous state. If a perturbation pushes the system out of synchrony at such an intermediate value, in a chaotic regime characterized by $\lambda_{1}>0$, would it be possible to enhance synchronization and stability by controlling a small subset of nodes? In the following we will give a positive answer to this question, by exploring the dynamics of the system at $K \approx 816 \mathrm{MW}$ $(K \approx 729 \mathrm{MW})$ for $P^{\mathrm{G}}\left(P^{\mathrm{R}}\right)$, where deterministic chaos is present, i.e., $\lambda_{1}=0.0187\left(\lambda_{1}=0.096\right)$, and the system is not perfectly frequency synchronized: $\langle\Delta \omega\rangle_{t} \approx 0.34 \mathrm{~Hz}$ $\left(\langle\Delta \omega\rangle_{t} \approx 0.91 \mathrm{~Hz}\right)$, modeling a strongly perturbed power grid. From the average frequency profile shown in panel c (panel d) for $P^{\mathrm{G}}\left(P^{\mathrm{R}}\right)$, we can see that a major part of the power grid is frequency synchronized while few nodes have a significant frequency deviation and are identified as solitary states: 9 nodes for $P^{\mathrm{G}}, 11$ nodes for $P^{\mathrm{R}}$. (Note that the three solitary nodes $i=1,2,3$ can only be resolved in the blownup inset.) Solitary nodes oscillate with their own average frequency and do not resynchronize in a self-organized way at a given coupling strength, being thus critical for desynchronization. Note that the solitary nodes include those with the largest $\xi_{i}$, but not only those. In order to visualize this scenario in terms of running power grids, we can resort to the infinite bus model, commonly used in engineering literature to analyse the return to synchrony after a frequency perturbation at a node: the standard analysis neglects the back-reaction of the dynamics at node $i$ on the other nodes and keeps them fixed, i.e. $\ddot{\theta}_{i}+\alpha \dot{\theta}_{i}=\frac{P_{i}-K \sin \left(\theta_{i}\right)}{I_{i} \omega_{\mathrm{G}}}$. When decoupling this system $(K=0)$ the oscillator rotates freely with frequency $P_{i} /\left(\alpha I_{i} \omega_{\mathrm{G}}\right)$. When the coupling is switched on, this limit cycle persists, and in the absence of losses its average frequency stays close to $P_{i} /\left(\alpha I_{i} \omega_{\mathrm{G}}\right)$. This might be seen as a simple model for solitary states, where the infinite bus represents the remaining synchronous component. If they occur in a running grid the solitary node is normally switched off as soon as its frequency falls outside a certain range around the nominal value.

Control application. In the following we propose to apply the control term only to a small subset of nodes selected according to their dynamical properties. In particular we aim at designing a control strategy based on the dynamical properties of the system that allows us to attain synchronization by controlling few nodes only, thus speeding up the efficiency of control. In order to find such a set, different control strategies are proposed in the following: (i) the first

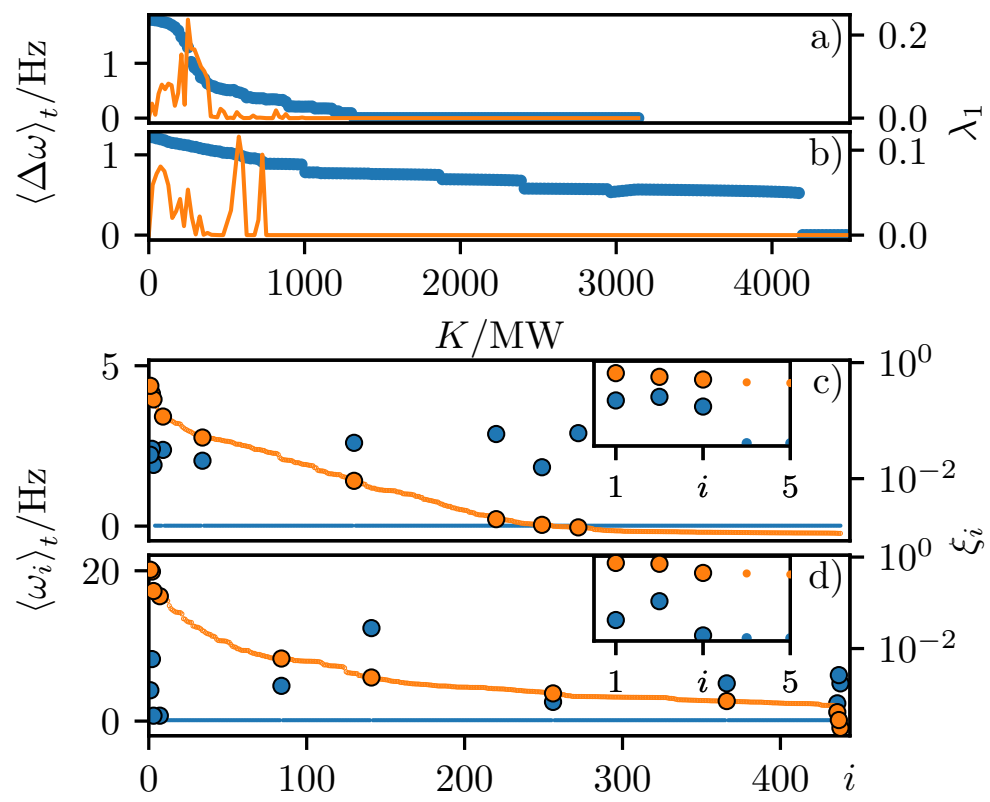

FIG. 2. Time averaged standard frequency deviation $\langle\Delta \omega\rangle_{t}$ (blue dots) and maximum Lyapunov exponent $\lambda_{1}$ (orange line) versus coupling strength $K$ for the bimodal Gaussian $P^{\mathrm{G}}$ (a) and for the real-world distribution $P^{\mathrm{R}}(\mathrm{b})$. Time averaged phase velocity profile $\left\langle\omega_{i}\right\rangle_{t}$ (blue dots) and Lyapunov vector components $\xi_{i}$ (orange dots) versus node index $i$ for the bimodal Gaussian at $K \approx 819 \mathrm{MW}$ (c) and for the real-world distribution at $K \approx 729 \mathrm{MW}(\mathrm{d})$. Data are ordered in descending order of $\xi_{i}$. The insets show a zoom for small $i$. Large filled circles mark solitary nodes. For $P^{\mathrm{G}} 0 \leq K \leq 3142 \mathrm{MW}$ in steps of $\Delta K \approx 21 \mathrm{MW}$ with $\alpha=5 / 6 \mathrm{~s}^{-1}$. For $P^{\mathrm{R}} 0 \leq K \leq 4500 \mathrm{MW}$ in steps of $\Delta K \approx 25 \mathrm{MW}$ with $\alpha=2 \mathrm{~s}^{-1}$. Other parameters: $I_{i}=I=40 \cdot 10^{3} \mathrm{~kg} \mathrm{~m}^{2}, \omega_{G}=50 \mathrm{~Hz}, T_{A}=400 \mathrm{~s}$, time averages over $100 \mathrm{~s}$.

strategy takes into consideration all solitary nodes, sorted in descending order of $\xi_{i}$; (ii) the second strategy orders the solitary nodes by their absolute average frequency $\left|\langle\omega\rangle_{t}\right|$; (iii) the third strategy consider all nodes, not only solitary ones, randomly picked. The outcome of the different strategies is shown in Fig. 3(a)-(c) and (d)-(f) for the bimodal Gaussian distribution $P^{\mathrm{G}}$ and the real-world distribution $P^{\mathrm{R}}$, respectively. First of all, strategy (i) is able to achieve stability if just one node is controlled, and frequency synchronization if the number of controlled solitary nodes is sufficiently large: 8 controlled nodes for both $P^{\mathrm{G}}$ and $P^{\mathrm{R}}$. Strategy (ii) requires 4 controlled nodes for stabilization and 8 for synchronization in case of $P^{\mathrm{G}}$, and one controlled node for stabilization and 9 nodes for synchronization in case of $P^{\mathrm{R}}$. The third strategy is not able to frequency-synchronize and stabilize, it can at most mitigate to some extent the desynchronization and the instability. For the given setup, strategy (i) is the best choice. It stabilizes the system by controlling just one node and forces the solitary nodes, whose frequency deviates most, to frequency-synchronize. This strategy is particularly efficient since the Lyapunov vector is re-calculated every time when an additional solitary node is controlled, thus taking 


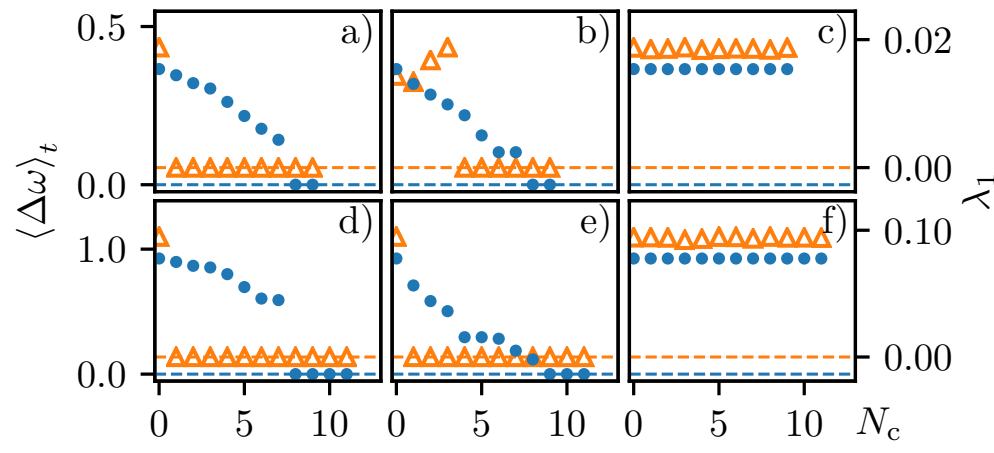

FIG. 3. Efficiency of time-delayed feedback control: time averaged frequency deviation $\langle\Delta \omega\rangle_{t}$ (blue dots) and maximum Lyapunov exponent $\lambda_{1}$ (orange triangles) vs. number of controlled nodes $N_{\mathrm{c}}$ following different control strategies: (a), (d) solitary nodes sorted in descending order of $\xi_{i}$. (b), (e) solitary nodes sorted in descending order of $\left|\left\langle\omega_{i}\right\rangle_{t}\right|$. (c), (f) randomly picked nodes. Panels (a)-(c) correspond to the distribution $P^{\mathrm{G}},(\mathrm{d})-(\mathrm{f})$ to $P^{\mathrm{R}}$. The dashed lines mark $\langle\Delta \omega\rangle_{t}=0, \lambda_{1}=0$. Control acts for a duration of 40 seconds and is then turned off; delay time $\tau=4 \mathrm{~s}$, feedback gain $g=1$, other parameters as in Fig. 2, time averages over $80 \mathrm{~s}$.

into account the interplay between solitary states and emerging instabilities.

In [25] numerical evidence was given that dead ends and dead trees undermine basin stability of nodes in Kuramoto power grid networks, which means that the basin of attraction of the frequency synchronized solution for single nodes tends to be small if a node is placed at a dead end, thus making such nodes hard to synchronize. Indeed, in the case of the bimodal Gaussian distribution $P^{\mathrm{G}}$, all the identified solitary nodes belong to a dead tree (see Fig. 4a). However, this trend cannot be observed for the real-world distribution $P^{\mathrm{R}}$, where just 3 of the 11 solitary nodes belong to a dead tree (see Fig. 4b) and dead trees do not correspond to the most unstable nodes. In general we have observed that the most unstable solitary nodes, for $P^{\mathrm{G}}$, are dead ends adjacent to well connected nodes, whereas for $P^{\mathrm{R}}$ they are nodes with natural frequency $P_{i}>4 \Delta P$, where $\Delta P$ is the standard deviation of the distribution. The discrepancy between the two cases can be explained if, starting from $P^{\mathrm{G}}$, we arbitrarily add $4 \Delta P$ to the net power ( $\widehat{=}$ inherent frequency) of a nonsolitary node $k$. This altered node then becomes solitary and causes other adjacent nodes to become solitary, some of them belonging to dead trees. If we control all the newly emerged solitary dead trees, the system does not synchronize and the dynamics of node $k$ is almost unchanged (Fig. $4 \mathrm{c}$ ), whereas we can achieve synchronization via controlling node $k$ only (Fig. 4d). This means that dead trees are fundamental in determining the power grid stability whenever the frequency distribution does not contain fat tails or $e x$ treme events, which is the case for $P^{\mathrm{G}}$; for the real-world distribution $P^{\mathrm{R}}$, however, nodes with significant frequency difference are common and the stability is undermined by these nodes rather than by dead trees.
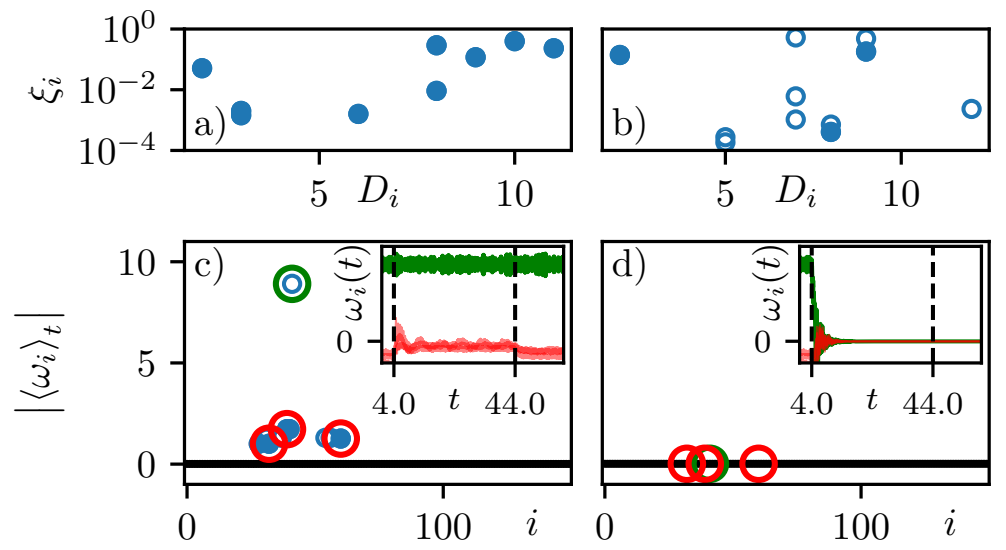

FIG. 4. Source of solitary nodes: Lyapunov vector components $\xi_{i}$ versus maximum neighborhood degree $D_{i}$ for (a) $P^{\mathrm{G}}$, (b) $P^{\mathrm{R}}$. Only solitary nodes are shown, and filled circles identify nodes which belong to dead trees. (c),(d): Absolute time-averaged frequency $\left|\left\langle\omega_{i}\right\rangle_{t}\right|$ versus node index $i$ for $P^{\mathrm{G}}$, where $4 \Delta P$ is added to the inherent frequency of an arbitrary non-solitary node $k$ (green circle). In (c) dead-tree nodes (red circles) adjacent to $k$ are controlled and in (d) $k$ is controlled. Black dots are synchronized nodes, blue symbols are solitary nodes. Nodes belonging to a dead tree are marked by filled symbols. The instantaneous frequencies $\omega_{i}(t)$ of green and red nodes versus time are shown in the insets. Vertical dashed lines mark activation and deactivation of control. Parameters as in Figs.2 and 3, time averages over 80 s.

Conclusions. In conclusion, we have proposed a timedelayed feedback control scheme to restore frequency synchronization and stability of the power grid after perturbations. To this purpose we have studied the Kuramoto model with inertia in the presence of a bimodal distribution of generator and load power, which leads to a fully frequency synchronized, stable network for large transmission capacities, both when an artificial bimodal Gaussian distribution of power generation and consumption, or a distribution adapted from the real power grid of Germany is used. We have focussed on the operating regime of intermediate transmission capacities where the steady state is dynamically Lyapunov unstable and the partial synchronization pattern is characterized by a number of solitary nodes whose timeaveraged frequency deviates from the mean frequency of all other nodes.

We have shown that stability and synchronization can be enhanced by time-delayed feedback control in the regime of intermediate transmission capacities, where large perturbations cause asynchronous and unstable power grid operation. Applying delayed feedback to a small subset of nodes, namely the set of solitary nodes, we have demonstrated that frequency synchronization and stability can be restored in a short time, and that these states persist even if control is turned off. Different control strategies were tested. We conclude that the best strategy is to control the most unstable solitary nodes, characterized by the largest Lyapunov vector components. Solitary nodes exhibit independent dynamics, 
giving rise to low-dimensional chaos that turns into highdimensional quasi-periodic motion when the most unstable node is controlled, until the synchronization is achieved [45]. Therefore, due to their independence, the set of controlled nodes cannot be much smaller than the number of solitary nodes. The proposed fast-acting control method might offer an interesting approach to cure disturbances in real-world power grids. Data from the German high voltage transmission grid were used as a proof of principle.

*simona.olmi@inria.fr

[1] M. G. Rosenblum, and J. Kurths, Synchronization: a universal concept in nonlinear sciences (Cambridge University Press, Cambridge, 2001).

[2] S. Boccaletti, A. N. Pisarchik, C. I. del Genio, and A. Amann, Synchronization: From Coupled Systems to Complex Networks (Cambridge University Press, Cambridge, 2018).

[3] C. van Vreeswijk, Phys. Rev. E 54, 5522 (1996).

[4] C. van Vreeswijk, Phys. Rev. Lett. 84, 5110 (2000).

[5] S. H. Strogatz, Nature 410, 268 (2001).

[6] Y. Maistrenko, B. Penkovsky, and M. Rosenblum, Phys. Rev. E 89, 060901 (2014).

[7] P. Jaros, S. Brezetsky, R. Levchenko, D. Dudkowski, T. Kapitaniak, and Y. Maistrenko, Chaos 28, 011103 (2018).

[8] P. Milan, M. Wächter, and J. Peinke, Phys. Rev. Lett. 110, 138701 (2013).

[9] D. Heide, L. von Bremen, M. Greiner, C. Hoffmann, M. Speckmann, and S. Bofinger, Renewable Energy 35, 2483 (2010).

[10] D. Heide, M. Greiner, L. von Bremen, and C. Hoffmann, Renew. Energy 36, 2515 (2011).

[11] Union for the Coordination of Transmission of Electricity (UCTE), "Final Report System Disturbance on 4 November $2006 "$ ".

[12] A. E. Motter and Y. C. Lai, Phys. Rev. E 66, 065102 (2002).

[13] S. V. Buldyrev, R. Parshani, G. Paul, H. Eugene Stanley, and H. Shlomo, Nature 464, 1025 (2010).

[14] B. Schäfer, C. Beck, K. Aihara, D. Witthaut, and M. Timme, Nature Energy 3, 119 (2018).

[15] I. Simonsen, L. Buzna, K. Peters, S. Bornholdt, and D. Helbing, Phys. Rev. Lett. 100, 218701 (2008).

[16] H. D. Chiang, Direct Methods for Stability Analysis of Electric Power Systems: Theoretical Foundation, BCU Methodologies, and Applications (John Wiley \& Sons, 2010).

[17] M. Anvari, G. Lohmann, M. Waechter, P. Milan, E. Lorenz, D. Heinemann, M. Reza Rahimi Tabar, and J. Peinke, New J. Phys. 18, 063027 (2016).

[18] B. Schäfer, M. Matthiae, M. Timme, and D. Witthaut, New J. Phys. 17, 015002 (2015).

[19] P. J. Menck, J. Heitzig, N. Marwan, and J. Kurths, Nat. Phys. 9, 89 (2013).

[20] M. Rohden, A. Sorge, M. Timme, and D. Witthaut, Phys. Rev. Lett. 109, 064101 (2012).

[21] D. Witthaut and M. Timme, New J. Phys. 14, 083036 (2012).

[22] E. B. T. Tchuisseu, D. Gomila, P. Colet, D. Witthaut, M. Timme, and B. Schäfer, New J. Phys. 20, 083005 (2018).
[23] A. E. Motter, S. A. Myers, M. Anghel, and T. Nishikawa, Nat. Phys. 9, 191 (2013).

[24] F. Dörfler, M. Chertkov, and F. Bullo, Proc. Natl. Acad. Sci. U.S.A. 110, 2005 (2013).

[25] P.J. Menck, J. Heitzig, J. Kurths, and H. J. Schellnhuber, Nat. Commun. 5, 3969 (2014).

[26] D. Witthaut, M. Rohden, X. Zhang, S. Hallerberg, and M. Timme, Phys. Rev. Lett. 116, 138701 (2016).

[27] K. Pyragas, Phys. Lett. A 170, 421 (1992).

[28] E. Schöll and H. G. Schuster, eds., Handbook of Chaos Control (Wiley-VCH, Weinheim, 2008) second completely revised and enlarged edition.

[29] G. Filatrella, A. H. Nielsen, and N. F. Pedersen, Eur. Phys. J. B 61,485 (2008).

[30] J. Machowski, J. Bialek, and J. R. Bumby, Power System Dynamics: Stability and Control, 2nd ed. (John Wiley \& Sons, 2008)

[31] S. H. Horowitz and A. G. Phadke, Power system relaying (John Wiley \& Sons, 2008).

[32] J. Egerer, Open Source Electricity Model for Germany (ELMOD-DE), Tech. Rep. (Deutsches Institut fur Wirtschaftsforschung (DIW), 2016).

[33] M. Rohden, A. Sorge, D. Witthaut, M. Timme, Chaos: An Interdisciplinary Journal of Nonlinear Science, 24(1), 013123 (2014).

[34] S. Lozano, L. Buzna, and A. Diáz-Guilera, Eur. Phys. J. B 85, $231(2012)$

[35] S. Olmi, A. Navas, S. Boccaletti, and A. Torcini, Phys. Rev. E 90, 042905 (2014).

[36] S. Olmi and A. Torcini, in Control of Self-Organizing Nonlinear Systems, edited by E. Schöll, S. H. L. Klapp, and P. Hövel (Springer International Publishing, 2016) Chap. 2, pp.25-45.

[37] L. Tumash, S. Olmi, E. Schöll, EPL 123, 20001 (2018).

[38] G. Benettin, L. Galgani, A. Giorgilli, and J.-M. Strelcyn, Meccanica 15, 9 (1980).

[39] A priori the relationship between Lyapunov vectors and eigenvectors of the Jacobian matrix is not obvious, since Lyapunov vectors are in general not identical with the local principal expanding and contracting directions, i.e., the eigenvectors of the Jacobian. In fact, while the latter require only local knowledge of the system, the Lyapunov vectors are influenced by all Jacobians along a trajectory, thus representing a measure of the instability of the dynamics of the system, not related to any particular fixed point or initial stable solution. Therefore the calculation of Lyapunov vector with respect to the eigenvectors of the Jacobian matrix is more powerful since the former one is not necessarily restricted to the synchronized solution or to a particular linearisation point.

[40] P. Kundur, N. J. Balu, and M. G. Lauby, Power System Stability and Control, (McGraw-Hill, 1994).

[41] H. Bevrani, Robust power system frequency control, (New York: Springer, 2009).

[42] G. Rinaldi, M. Cucuzzella, and A. Ferrara, IEEE Control Systems Letters 1(2), 215-220 (2017).

[43] H. Okuno, M. Kawakita, Electrical Engineering in Japan 156, 7-12 (2006)

[44] E. D. Dongmo, P. Colet, P. Woafo, The European Physical Journal B 90, 6 (2017).

[45] H. Taher, S. Olmi, E. Schöll, Phys. Rev. E 100, 062306 (2019). 